\title{
INVESTIGATION OF PHONON SUPPRESSION BY NANOSTRUCTURING AND DOPING IN THERMOELECTRIC HALF-HEUSLER MATERIALS
}

\author{
1,2Miloš FEJERČÁK, ${ }^{1,2}$ Karel SAKSL, 'Zuzana MOLČANOVÁ, ${ }^{1,3}$ Katarína ŠUL'OVÁ, \\ ${ }^{2}$ Michaela ŠULIKOVÁ, ${ }^{4}$ Margarita RUSSINA, ${ }^{4}$ Veronika GRZIMEK, ${ }^{4}$ Gerrit GUENTHER \\ ${ }^{1}$ Institute of Materials Research, Slovak academy of sciences, Košice, Slovakia, EU, imr.saske.sk \\ ${ }^{2}$ Pavol Jozef Šafárik University, Košice, Slovakia, EU, upjs.sk \\ ${ }^{3}$ Faculty of Materials Metallurgy and Recycling, Technical University of Košice, Slovakia, EU, fmmr.tuke.sk \\ ${ }^{4}$ Helmholtz Zentrum Berlin, Berlin, Germany, EU, helmholtz-berlin.de
}

https://doi.org/10.37904/metal.2019.754

\begin{abstract}
We live in the age when humanity finds itself on the edge of energy crisis, fossil fuels are consumed and our energy consumption rises every year. One solution would be to obtain energy from renewable sources and to minimize the losses of energy produced, e.g. reuse the waste heat. Thermoelectric materials can convert heat directly and reversibly into electricity and allow therefore to use waste thermal energy more efficiently. Their benefits include the absence of moving parts, quiet operation, reliability, durability, and the fact that they do not produce any polluting emissions, so we can use them in a wide range of applications and they are also attractive from an environmental point of view. Half-Heusler alloys belong to one of the most promising thermoelectric materials composed of relatively non-toxic and abundant elements, with highest ZT $=1.5$ at $700 \mathrm{~K}$ for $\mathrm{Zr}_{0.25} \mathrm{Hf}_{0.25} \mathrm{Ti}_{0.5} \mathrm{Ni}_{1} \mathrm{Sn}_{0.998} \mathrm{Sb}_{0.002}$ at\% alloy. In our study we try to improve the thermoelectric performance of this alloy by doping it with semiconducting dispersion phase - $\beta-\mathrm{FeSi}_{2}$, which should reduce the thermal conductivity of the origin alloy. Since thermal conductivity depends to large extent on the propagation of phonons we have investigated how the nanostructuring of the samples by means of ball milling and doping impact the phonon behavior. For this purpose we have conducted inelastic neutron scattering experiments using the time-of flight spectrometer NEAT at Helmholtz Zentrum Berlin. In this paper are presented results of our study demonstrating the effect of phonon suppression by nanostructuring and doping in thermoelectric half-Heusler alloys.
\end{abstract}

Keywords: Thermoelectric materials, phonons, half-Heusler alloys, inelastic neutron scattering

\section{INTRODUCTION}

Thermoelectric materials have been extensively studied because of their huge potential in waste heat recovery and refrigeration industry [1]. They can convert temperature gradient in to electricity and vice versa. The efficiency of this process is summarized by the dimensionless figure of merit

$Z T=\frac{s^{2} \sigma}{\kappa} T$

where:
$S$ - the Seebeck coefficient $(\mu \mathrm{V} / \mathrm{K})$,
$\sigma$ - the electrical conductivity $(\mathrm{S} / \mathrm{m})$,
$\kappa$ - the thermal conductivity $\left(\mathrm{W} \mathrm{m}^{-1} \mathrm{~K}^{-1}\right)$,
$T$ - the absolute temperature $(\mathrm{K})$.

From equation (1) is obvious that for increase of $Z T$ we have either to increase the numerator $S^{2} \sigma$ (the power factor) or to decrease $\kappa$. All three parameters $S, \sigma, k$ are strongly connected. The $\sigma$ parameter depends on number of the free charge carriers. The thermal conductivity $K$ is combination of two terms - electrical $\kappa_{e}$ (heat 
transported by charge carriers) and lattice thermal conductivity $K_{L}$, where heat is transported through crystal lattice by form of lattice vibrations - phonons. The $\kappa_{e}$ parameter is interconnected to the $\sigma$ by Wiedemann-Franz law [2] and therefore in praxis the reduction of $K$ is realized only by decreasing $K_{L}$. From the kinetic theory, the lattice thermal conductivity can be estimated as

$\kappa_{L}=\frac{1}{3} C v_{m} l$

where:

$$
\begin{aligned}
& C \text { - the lattice specific heat }(\mathrm{J} / \mathrm{K}), \\
& v_{m} \text { - the mean sound velocity }(\mathrm{m} / \mathrm{s}), \\
& I \text { - the mean free path of the phonons }(\mathrm{m}) \text {. }
\end{aligned}
$$

There are several approaches to reduce $\kappa_{L}$ by means of reducing I (scattering of the phonon waves): nanostructuring [3], alloying [4], and guest atom inclusion [5]. Particularly powerful method used for studying phonons is inelastic neutron scattering.

The aim of the presented work was to develop a new thermoelectric material with high conversion efficiency, which would consist of non-toxic, inexpensive and affordable elements. Therefore, we decided to produce a half-Heusler $(\mathrm{HH})$ alloy: $Z \mathrm{r}_{0.25} \mathrm{Hf}_{0.25} \mathrm{Ti}_{0.5} \mathrm{Ni}_{1} \mathrm{Sn}_{0.998} \mathrm{Sb}_{0.002}$ at.\%, which achieves $Z T_{\max }=1.5$ at $700 \mathrm{~K}$ [6]. To decrease lattice thermal conductivity we introduce nanostructuring of the sample by means of high energy ball milling and spark plasma sintering (sample $\mathrm{HHO}$ ). For further lowering of $\kappa_{L}$ we have doped this alloy with a semiconducting dispersion phase $\beta-\mathrm{FeSi}_{2}$ in the form of $2 \mathrm{vol} \%$ (sample $\mathrm{HH} 1$ ). Inelastic neutron scattering experiments were performed to understand lattice dynamics - phonon behavior of nanostructuring and doping effects on $\mathrm{HH}$ samples. To compare temperature dependent phonon density of states (DOS) of ball milled $\mathrm{HHO}$ sample and doped sample $\mathrm{HH} 1$, we measured also the alloy in well relaxed state, prepared by arc-melting and annealed in furnace at high temperature (sample $\mathrm{HHOO}$ ). For better clarity, chemical compositions and preparation processes of each sample are summarized in Table 1.

Table 1 Thermoelectric $\mathrm{HH}$ samples

\begin{tabular}{|c|c|c|}
\hline Name & Chemical composition & Preparation methods \\
\hline $\mathrm{HH} 00$ & $\mathrm{Zr}_{0.25} \mathrm{Hf}_{0.25} \mathrm{Ti}_{0.5} \mathrm{Ni}_{1} \mathrm{Sn}_{0.998} \mathrm{Sb}_{0.002}(\mathrm{at} \%)$ & $\begin{array}{c}\text { Arc-melting: } 3 \mathrm{x} \text {, Annealing } 3 \mathrm{~h} \text { at } \\
800{ }^{\circ} \mathrm{C} \text { in protective atmosphere }\end{array}$ \\
\hline $\mathrm{HH} 0$ & $\mathrm{Zr}_{0.25} \mathrm{Hf}_{0.25} \mathrm{Ti}_{0.5} \mathrm{Ni}_{1} \mathrm{Sn}_{0.998} \mathrm{Sb}_{0.002}(\mathrm{at} \%)$ & $\begin{array}{c}\text { Arc-melting: } 3 \mathrm{x}, \text { Ball milling: } 1 \mathrm{~h} \text { in } \\
\text { protective atmosphere, SPS }\end{array}$ \\
\hline $\mathrm{HH} 1$ & $\mathrm{Zr}_{0.25} \mathrm{Hf}_{0.25} \mathrm{Ti}_{0.5} \mathrm{Ni}_{1} \mathrm{Sn}_{0.998} \mathrm{Sb}_{0.002}(\mathrm{at} \%)+2 \mathrm{vol} \% \beta-\mathrm{FeSi}_{2}$ & $\begin{array}{c}\text { Arc-melting: } 3 \mathrm{x}, \text { Ball milling: } 1 \mathrm{~h} \text { in } \\
\text { protective atmosphere, SPS }\end{array}$ \\
\hline
\end{tabular}

\section{MATERIALS PREPARATION}

All HH samples were prepared from elements of high purity: $\mathrm{Zr}(99.2 \%), \mathrm{Hf}(99.7 \%), \mathrm{Ti}(99.99 \%), \mathrm{Ni}(99.98 \%)$, Sn (99.9\%), Sb (99.9999\%), Fe (99.98\%), Si (99.9999\%). After proper weighting, samples were arc-melted, at least 3 times to ensure homogeneity, in the Edmund Bühler - Compact Arc Melter MAM-1 using maximum power of the device. Additionally, $\mathrm{HH} 00$ was annealed for 3 hours at $800{ }^{\circ} \mathrm{C}$ in tube furnace in order to reduce the residual stresses formed by rapid cooling in arc-melting process. $\mathrm{HH} 0$ and $\mathrm{HH} 1$ ingots were firstly crashed, then pre-milled in vibration mill and finally ball milled under Ar atmosphere for 1 hour at 200 RPM in planetary ball mill - FRITSCH PULVERISETTE 6. Prepared powders were compacted by spark plasma sintering (SPS) at temperature $1473 \mathrm{~K}$ under pressure $16 \mathrm{kN}$ for 10 minutes using $20 \mathrm{~mm}$ graphite dies in - SPS HP D10-SD. Obtained compacts (pellets) were then cleaned from graphite and polished. 


\section{NEUTRON SCATTERING EXPERIMENTS}

In order to determine thermal dynamics of the $\mathrm{HH}$ samples, inelastic neutron scattering (INS) experiments were performed. All $3 \mathrm{HH}$ samples were measured at the NEAT time-of-flight (TOF) spectrometer - V3 beamline located at Helmholtz-Zentrum Berlin (HZB). HZB is operating a swimming-pool fission reactor BER II. with cold source of $10 \mathrm{MW}$ power.

\subsection{NEAT Spectrometer}

NEAT is a chopper system TOF spectrometer. Neutrons are generated in cold source reactor and then travel a long distance of $64 \mathrm{~m}$ to the sample. On their way through neutron guide they pass seven rotating discs choppers with small openings, which function is to cut neutrons of desired wavelength $\left(\lambda_{0}\right)$ from continuous energy spectrum of the reactor. Second important function of choppers is, that they create a well-defined time pulses of neutrons, what is necessary for the TOF technique. Monochromatic neutron pulses then hit the sample and are scattered to detector. The sample to detectors distance is constant $3 \mathrm{~m}$. NEAT is composed of $416{ }^{3+} \mathrm{He}$ radially placed detectors covering scattering angles from $142^{\circ}$ to $-82^{\circ}$ [7]. With known distances between pulse creating chopper - sample - detectors and with fixed $\lambda_{0}$, we are now able to measure TOF of neutron scattered on the sample. Scattered neutrons arrive at the detectors at times determined by their scattered energies $E$. Therefore we can calculate neutron energy transfer $\hbar \omega=E_{0}-E$, which in our energy range represents the phonons. Given the angle between the incident $\left(\boldsymbol{k}_{\mathbf{0}}\right)$ and scattered neutron wave-vectors $(\boldsymbol{k})$, i.e. the scattering angle $\boldsymbol{\Phi}$, the wave-vector transfer $\boldsymbol{Q}=\boldsymbol{k}_{\mathbf{0}}-\boldsymbol{k}$ is readily calculated. Measuring the intensities of time and angular scattered neutrons we can calculate a neutron scattering function $S(Q, \omega)$ or more precisely dynamic structure factor [8].

\section{EXPERIMENTAL PART}

\subsection{Instrumental setup}

Each $\mathrm{HH}$ sample was loaded into a flat $\mathrm{Al}$ sample holder $(\mathrm{SH})$ and placed to cryostat installed inside in the detector chamber. Upper part of the $\mathrm{SH}$ was covered by $\mathrm{Cd}$ shielding in order to reduce $\mathrm{Al}$ intensity as much as it was possible. Because we want to use full cross section of the neutron beam at the sample $(2 \times 6 \mathrm{~cm})$, we decided to cut the disc samples along and place them on each other like „8“, to cover more space. Sample $\mathrm{HHOO}$ (not SPS-ed) in form of round ingot was not cut. All 3 samples: $\mathrm{HHO}, \mathrm{HH} 1$ and $\mathrm{HHOO}$ were measured at neutron wavelengths: 2, 3, and $5 \AA$ by temperatures: $100,200,300,400 \mathrm{~K}$. At the same conditions empty SH (empty can) and also Vanadium standard were measured.

\subsection{Data treatment}

For data processing we have used MANTID software. Best resolution of phonon branches we obtained by using $0.3 \mathrm{~nm}$ wavelength, so in further calculations only this energy data will be used. Because measured samples had different scattering intensity, samples $\mathrm{HH} 00$ and $\mathrm{HH} 1$ were normalized to weight of the sample $\mathrm{HHO}$.

\section{RESULTS AND DISCUSSION}

The first insight of nanostructuring effect we observe by investigation of elastic part of the spectra. In Figure 1, there are presented elastic neutron spectra of all the $3 \mathrm{HH}$ samples measured at the temperatures 100,200 , 300 and $400 \mathrm{~K}$. On spectra from the $\mathrm{HHO}$ and $\mathrm{HH} 1$ samples we do not observe Bragg reflection (200). Suppression of the (200) peak can be attributed to effective nanostructurization of the origin alloy caused by high energy ball milling process - samples $\mathrm{HHO}$ and $\mathrm{HH} 1$. 

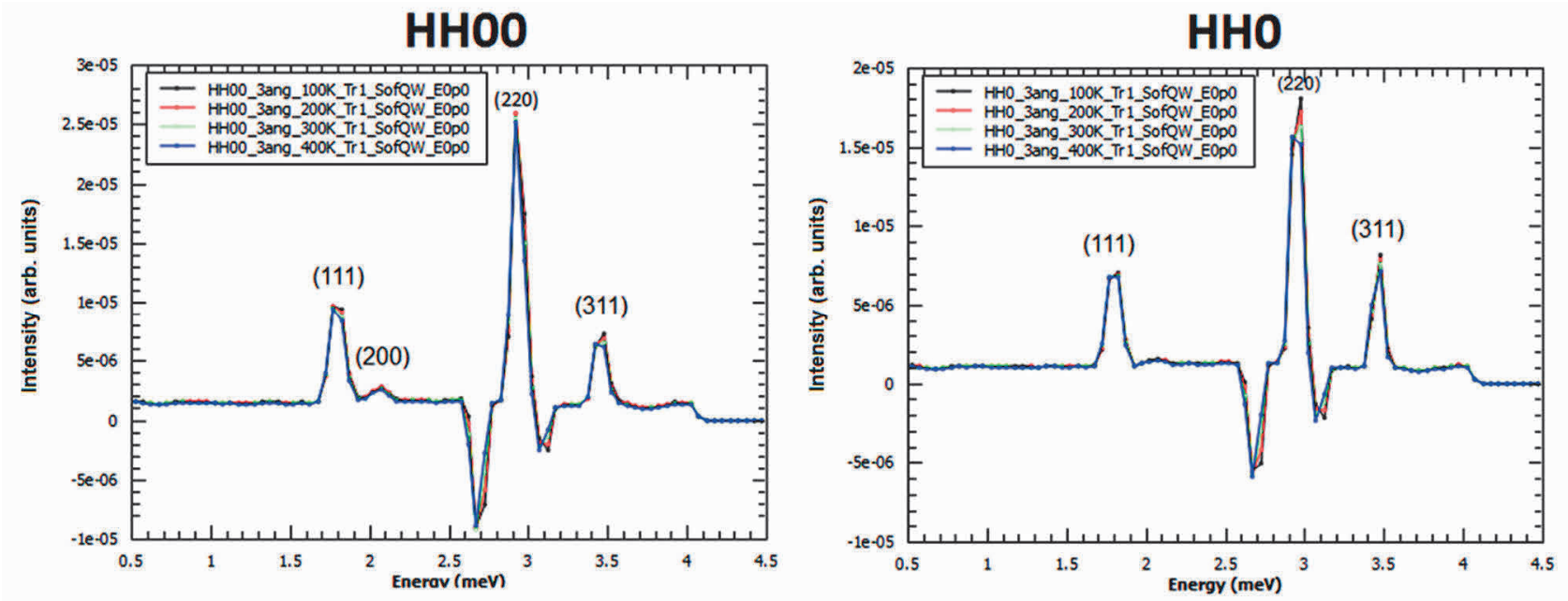

HH1

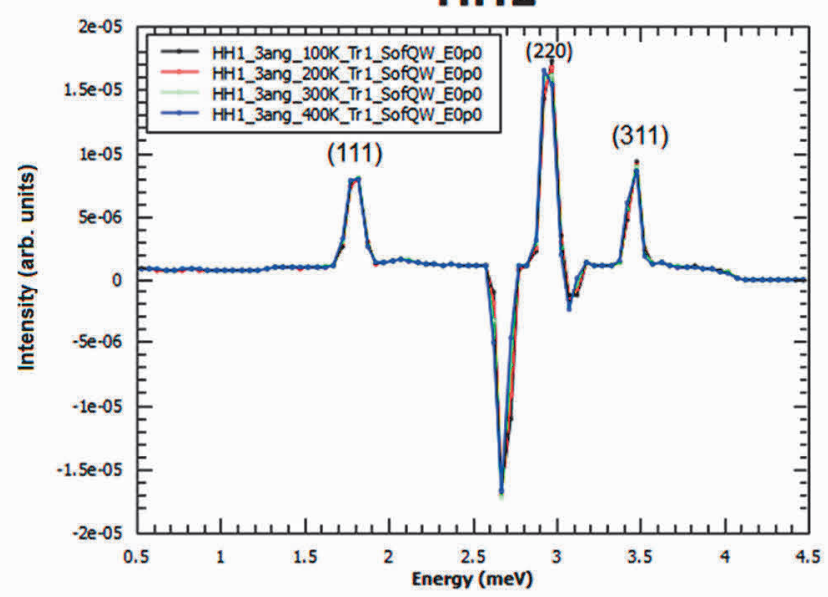

Figure 1 Elastic part of the neutron spectrum of $\mathrm{HH}$ samples
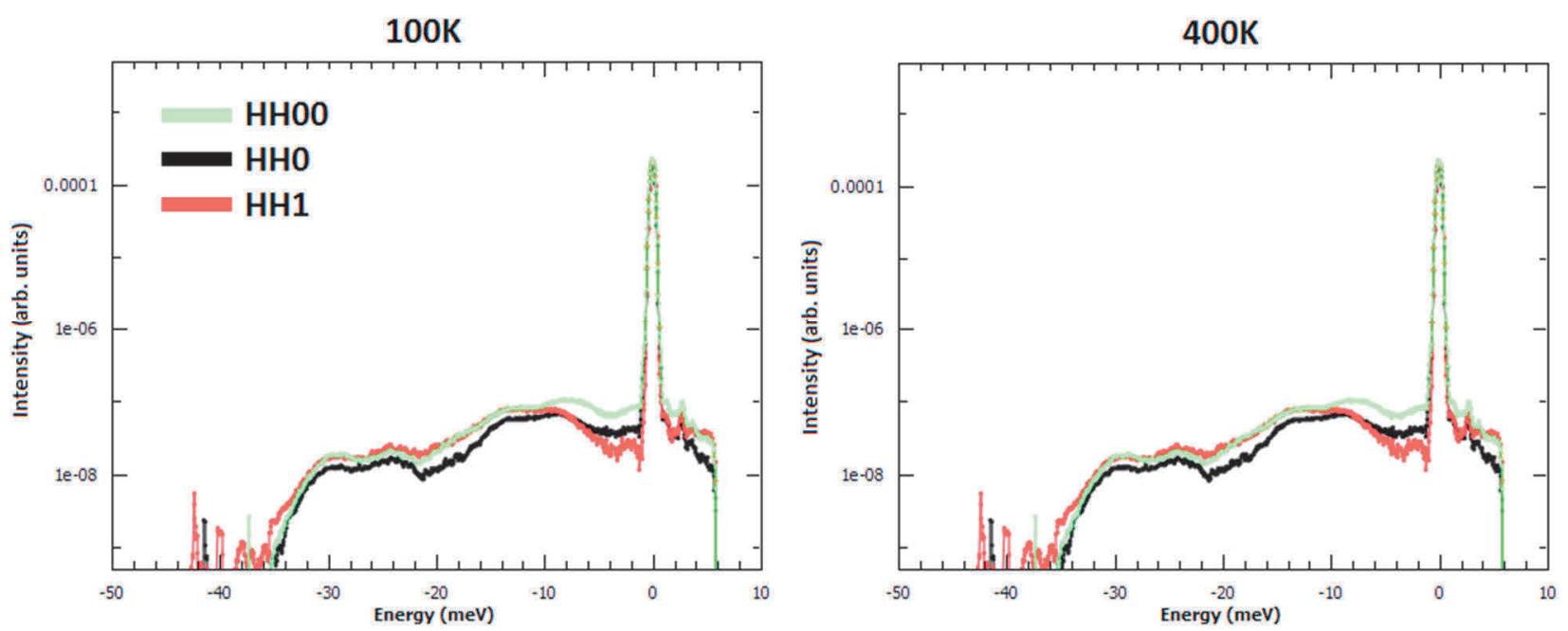

Figure 2 Comparison of inelastic neutron spectra for $3 \mathrm{HH}$ samples at the same temperatures

More information about the nanostructuring effect we obtain from inelastic part of spectra - phonon DOS. Figure 2 compares $\mathrm{HH}$ samples, normalized to the weight of $\mathrm{HHO}$, at the same temperatures. At each temperature is visible that the well annealed and relaxed $\mathrm{HHOO}$ sample exhibits higher intensity than 
HHO sample after ball milling and spark plasma sintering. Therefore, we can say that nanostructuring effectively suppresses phonons, since both samples have the same chemical composition, and they differ just in the method of preparation. Figure 2 shows phonon DOS only at $100 \mathrm{~K}$ and $400 \mathrm{~K}$. The graphs for $200 \mathrm{~K}$ and $300 \mathrm{~K}$ looks very similar. Overall intensity loss caused by nanostructuring is more visible on $S(Q, \omega)$ plots of samples $\mathrm{HHOO}$ and $\mathrm{HHO}$ measured at $400 \mathrm{~K}$, see Figure 3.

Sample $\mathrm{HH} 1$ cannot be directly compared to the origin $\mathrm{HH} 00$ sample because of the addition of $\beta-\mathrm{FeSi}_{2}$, which has different scattering length than pure $\mathrm{HH}$ alloy. However, the effect of doping with dispersion phase $\beta$-FeSi2 is clearly visible in region from 0 to $-10 \mathrm{meV}$, where sample $\mathrm{HH} 1$ has lower intensity then $\mathrm{HH} 0$ and so we can say that doping is effectively suppressing phonons at lower frequencies.

HHOO

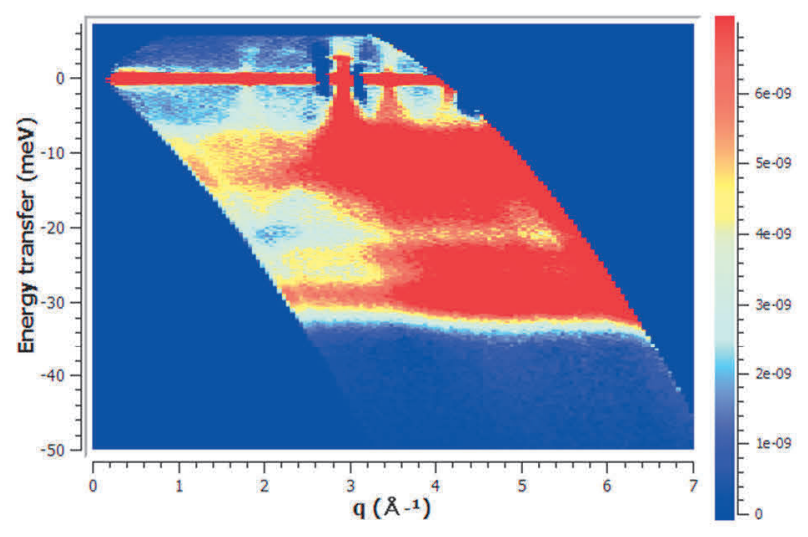

HH1

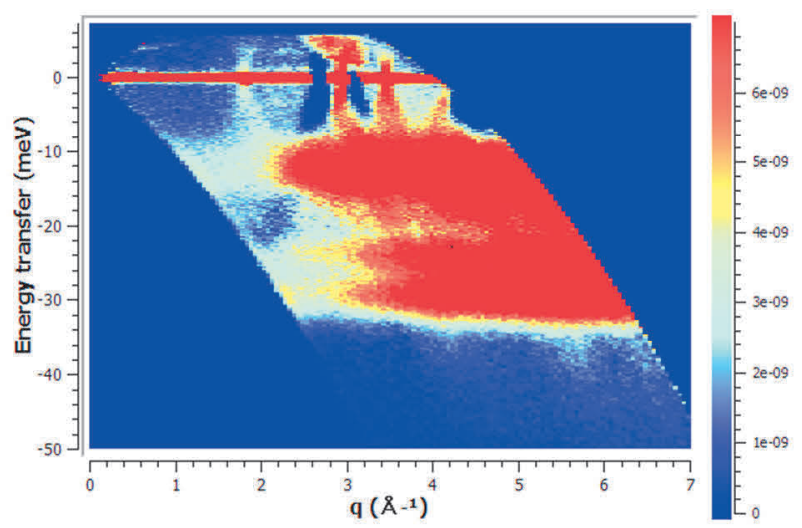

HHO

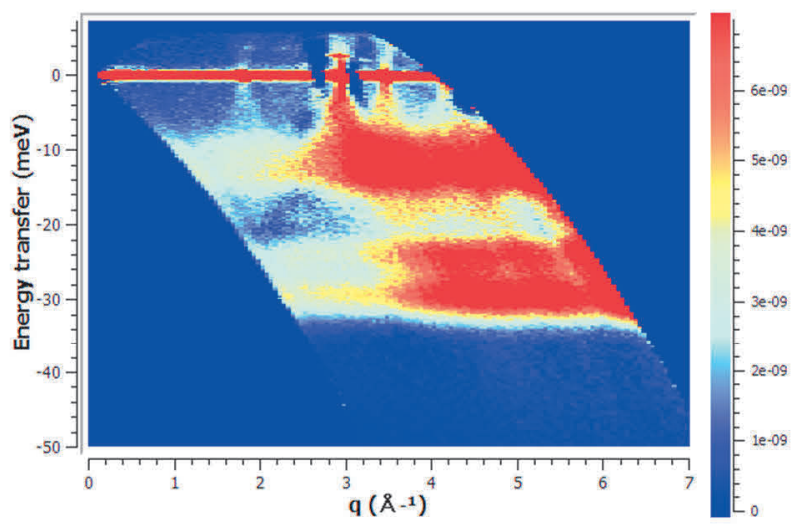

Figure $3 S(Q, \omega)$ plots of INS for $\mathrm{HH}$ samples measured at $400 \mathrm{~K}$ normalized to weight of $\mathrm{HH} 0$ sample

\section{CONCLUSION}

In here presented study, effects of high energy ball milling and doping with dispersion $\beta$-FeSi2 phase on halfHeusler thermoelectric alloys was investigated by means of inelastic neutron scattering experiments. The following results were obtained. Elastic part of INS spectra documents effective suppression of (200) Bragg peak caused by ball milling. Inelastic signals of the ball milled $\mathrm{HHO}$ sample shows overall intensity decrease compared to relaxed alloy $\mathrm{HH} 00$ in wide temperature range. The $S(Q, \omega)$ plots from the nanostructured samples show significantly lower intensity what confirm effective phonon suppression. The dispersion $\beta-\mathrm{FeSi}_{2}$ inclusion effect is most pronounced in the lower frequencies below - $10 \mathrm{meV}$, where the HH1 sample shows further phonon suppression. These phonon suppression effects are in agreement with our thermal conductivity 
measurements of $\mathrm{HH}$ samples where sample $\mathrm{HH} 1$ shows significantly lower thermal conductivity than sample $\mathrm{HHO}$.

\section{ACKNOWLEDGEMENTS}

Here I would like to express my thanks to the TOF group from Helmholtz Zentrum Berlin firstly for the opportunity to work with them and then for lots of help by neutron experiments. Also I want to thank national scholarship program of the Slovak Republic which provides me funding for my 4 months

stage at HZB. This work was supported by the research grant projects APVV-15-0202, APVV-17-0008 and VEGA No. 2/0013/19. This work was realized within the framework of the project ,Research

Centre of Advanced Materials and Technologies for Recent and Future Applications "PROMATECH“, ITMS 26220220186, supported by the Operational Program "Research and Development" financed through the European Regional Development Fund.

\section{REFERENCES}

[1] SNYDER, G. J. and TOBERER, E. S. Complex thermoelectric materials. Nat. Mater. 2008. vol. 7, no. 2, pp. 105114.

[2] JOHNSON, M. and MAHAN, G. D. Mott's formula for the thermopower and the Wiedemann-Franz law. Phys.Rev. B, 1980. vol. 21, no. 10 , pp. 4423-4429.

[3] POUDEU, P. F. P., ANGELO, J. D., DOWNEY, A. D., SHORT, J. L., HOGAN, T. P. and KANATZIDIS, M. G. High thermoelectric figure of merit and nanostructuring in bulk p-type. Chem. Int. Ed. 2006. Na 1, pp. 3835-3839.

[4] KIMURA, Y. and ZAMA, A. Thermoelectric properties of $p$-type half-Heusler compound HfPtSn and improvement for high-performance by Ir and Co additions thermoelectric properties of $p$-type half-Heusler compound HfPtSn and improvement for high-performance by Ir and Co additions. Appl. Phys. Lett. 2010. pp. 172110-172113.

[5] NOLAS, G. S., COHN, J. L., SLACK, G. A. and SCHUJMAN, S. B. Semiconducting Ge clathrates: Promising candidates for thermoelectric applications Semiconducting Ge clathrates: Promising candidates for thermoelectric applications. Appl. Phys. Lett., 1998. vol. 178, pp. 1-4.

[6] SAKURADA, S. and SHUTOH, N. Effect of Ti substitution on the thermoelectric properties of ( $\mathrm{Zr}, \mathrm{Hf}) \mathrm{NiSn}$ halfHeusler compounds Effect of Ti substitution on the thermoelectric properties of $\mathrm{Zr}, \mathrm{Hf} \ldots$ NiSn half-Heusler compounds. Appl. Phys. Lett. 2005. vol. 082105, pp. 8-11.

[7] RUSSINA, M. et al. Upgrade project NEAT'2016 at Helmholtz Zentrum Berlin - What can be done on the medium power neutron source? Phys. B Phys. Condens. Matter. 2017. vol. 551, no. 2018, pp. 506-511.

[8] SQUIRES, G. L. Introduction to the Theory of Thermal Neutron Scattering. 3rd ed. Cambridge: Cambridge University Press, 2012. p. 260. 